\title{
LA IMPORTANCIA DE LAS INSTITUCIONES: ALGUNAS REFLEXIONES
}

\author{
Vittorio Corbo \\ Centro de Estudios Públicos
}

\begin{abstract}
RESUMEN: Con ocasión de cumplirse un cuarto de siglo de la autonomía constitucional del Banco Central de Chile, Vittorio Corbo, uno de sus ex presidentes, realiza un recuento de los logros institucionales y la estabilidad que este marco legal ha significado. A partir de este exitoso ejemplo, propone dos nuevas instituciones autónomas: un renovado Instituto Nacional de Estadísticas y una Comisión de Productividad.

Palabras Clave: Banco Central, institucionalidad, Instituto Nacional de Estadísticas, Comisión de Productividad.
\end{abstract}

\section{THE IMPORTANCE OF INSTITUTIONS: SOME THOUGHTS}

ABSTRACT: As the Central Bank of Chile completes a quarter of a century of constitutional autonomy, Vittorio Corbo, a former president of this institution, recalls the achievements of the Central Bank and the stability that it has created. He proposes two new independent agencies: a renovated Statistics Bureau and a Productivity Commission.

Keywords: Central Bank, institutional framework, Statistics Bureau, Productivity Commission.

Vittorio Corbo (Iquique, 1943). Economista de la Universidad de Chile. PhD en economía del MIT. Ha sido profesor en la Concordia University de Montreal, Canadá, de la P. Universidad Católica de Chile y de la Universidad de Chile. Presidente del Banco Central entre los años 2003 y 2007. Consultor internacional de instituciones como el Banco Interamericano de Desarrollo y del Fondo Monetario Internacional. Hoy es investigador asociado senior del Centro de Estudios Públicos (CEP) y director de empresas. Email: vcorbo@cepchile.cl

El autor agradece los valiosos comentarios de Agustín Hurtado y José Tessada. 


\section{INTRODUCCIÓN}

S e acaban de cumplir 25 años de autonomía del Banco Central de Chile y ésta es una ocasión para reflexionar sobre la importancia que ha tenido en la estabilidad de Chile y las lecciones que se pueden sacar de este desarrollo institucional para otras áreas de políticas públicas en el país. Existe un amplio consenso en que tal autonomía ha contribuido, junto con la regla fiscal, a fortalecer significativamente la institucionalidad macroeconómica y financiera del país. En particular, le ha permitido al Banco Central tomar decisiones técnicas independientes, orientadas a velar por la estabilidad de precios y de los pagos internos y externos. El logro de esta preciada estabilidad ha creado las bases para un crecimiento alto y sostenido que le ha permitido a Chile no sólo dar un salto en su ingreso per cápita, sino además en el bienestar de todos los chilenos. En los últimos 25 años también se ha avanzado en la institucionalidad fiscal, en la promoción de la competencia, en el desarrollo de la infraestructura y, en menor medida, en las políticas sociales. Logros en esas áreas también se han realizado en las economías avanzadas, donde destacan los casos de Australia, Canadá y Nueva Zelandia. Estos desarrollos institucionales han jugado un rol importante en el progreso de estos y otros países. Así, es cada vez más reconocido que la calidad de las instituciones es un determinante sustancial de las diferencias de crecimiento entre las naciones (véase, entre otros, Olson 1984 y Acemoglu y Robinson 2012).

Los países avanzados han extendido el desarrollo institucional a otras áreas importantes. En particular, las oficinas de estadísticas son, por lo general, organismos del Estado con una gran independencia de los poderes ejecutivo y legislativo, lo que facilita la producción de información confiable para toda la sociedad. Las estadísticas producidas por estas instituciones son muy útiles no sólo para el diseño y evaluación de políticas públicas, sino que también son un bien público para la sociedad como un todo. En los últimos años se ha ido incluso más allá, creando organismos independientes para evaluar y diseñar cambios de políticas orientados a aumentar el bienestar de la población. En esta dirección, Australia ha sido pionera en la creación de un ente autónomo encargado de analizar y proponer cambios de políticas para aumentar la productividad y el crecimiento del país, con debida atención a aspectos medioambientales y territoriales. La Comisión de Productividad de 
Australia analiza y formula opciones de políticas públicas orientadas a aumentar la productividad y el bienestar del país en las áreas solicitadas por el poder ejecutivo, y también en las que surgen de su propia iniciativa. La autonomía de esta institución facilita el cumplimiento de su mandato. Para este propósito cuenta con un equipo técnico permanente y altamente calificado, y un proceso de consulta ciudadana ad hoc para preparar reformas inclusivas que contribuyan a mejorar el bienestar de la población. La buena evaluación de esta experiencia llevó también a Nueva Zelandia a crear una Comisión de Productividad, con la asistencia técnica de Australia. La experiencia reciente de diseño y de economía política de reformas en Chile muestra que la creación de un ente autónomo que asista a los gobiernos y a la sociedad como un todo en el diseño de reformas orientadas a mejorar el bienestar agregado podría generar grandes beneficios.

El presente artículo está organizado de la siguiente forma: en la sección 2 abordo los alcances que la autonomía ha tenido en el funcionamiento y desarrollo institucional del Banco Central de Chile; en la sección 3, a la luz de la experiencia de autonomía del Banco Central, expongo las oportunidades para fortalecer la institucionalidad en Chile en las áreas estadísticas y productividad. Finalmente, en la sección 4, concluyo.

\section{LECCIONES DE LA EXPERIENCIA DE UN BANCO CENTRAL DE CHILE AUTÓNOMO}

La creación de un Banco Central autónomo tuvo su origen en la Ley Orgánica Constitucional del Banco Central de Chile (BCCh), Ley N. ${ }^{\circ} 18.840$ del 10 de octubre de 1989. Esta ley fijó al BCCh el claro objetivo de velar por la estabilidad de la moneda (interpretado por su consejo como estabilidad de precios) y de los pagos internos y externos del país. Para poder cumplir con estos objetivos, la ley le otorgó al BCCh poderes para tomar decisiones técnicas independientes en el ejercicio de sus funciones. Desde el punto de vista patrimonial, la ley le concedió un patrimonio propio y la capacidad de administrar sus finanzas con total independencia del gobierno central.

Parte importante de la exitosa labor del Banco Central de Chile, y que se puede extender a otras instituciones, radica en su autonomía y 
su gobierno corporativo transversal y de alto nivel técnico. El término "autonomía" alude a la necesidad de algunas instituciones de salvaguardar sus decisiones de influencias políticas indebidas, dotándolas de independencia política y operativa. Las instituciones autónomas deben contar también con un adecuado sistema de checks and balances y con un procedimiento adecuado de rendición de cuentas. Instituciones autónomas cumplen con tres tipos de independencia: financiera; en el nombramiento y permanencia de su cuerpo directivo; y en la toma de decisiones tanto de políticas como de personal y de operaciones.

El BCCh no está sujeto a la supervisión de la Contraloría General de la República o de la Superintendencia de Bancos e Instituciones Financieras. Tampoco es parte de la administración general del Estado. El BCCh es gobernado por su consejo y regido exclusivamente por sus propias normas, limitado por su Ley Orgánica Constitucional y por algunas cláusulas de la Ley de Bancos. Finalmente, se concede al BCCh la facultad de interpretación administrativa de sus decisiones, regulaciones, órdenes e instrucciones, limitada solamente por las atribuciones legales de los cuerpos judiciales.

El fortalecimiento de la institucionalidad del BCCh ha sido un proceso largo, en el cual han participado todos los consejos, los directivos y los funcionarios que han sido parte de la institución, especialmente desde el inicio de la autonomía. El primer consejo enfrentó no sólo el problema de poner en práctica la nueva institucionalidad, sino que también debió iniciar el proceso de reducción gradual de la inflación en una economía donde el producto efectivo superaba al producto potencial, lo que repercutía en una aceleración de la inflación y un aumento en el déficit en cuenta corriente de la balanza de pagos (Bianchi 2014). Los sucesivos consejos se concentraron en perfeccionar el diseño e instrumentalización de la política monetaria y la regulación macro del sistema financiero, logrando avances importantes en estas dos áreas. No obstante, los consejos del BCCh han tenido que enfrentar una serie de problemas adicionales: i) la compatibilización de una política monetaria restrictiva orientada a reducir gradualmente la inflación con abultados ingresos de capitales que dificultaban esta labor; ii) el problema de la deuda subordinada; iii) las dificultades derivadas de enfrentar los efectos de la crisis asiática con ajustes acotados en el tipo de cambio; iv) los problemas de control de riesgos y de gestión; v) la respuesta a la 
gran crisis financiera (GCF) desatada a partir de la quiebra de Lehman Brothers; y vi), en años recientes, los problemas asociados a las políticas monetarias acomodaticias de los países avanzados. Cabe destacar que, luego de la experiencia de la crisis asiática, el BCCh dio un paso decidido hacia la implementación de un esquema pleno de metas de inflación, que incluyó la flexibilidad cambiaria (Mishkin y Schmidt-Hebbel 2007). En paralelo, se prepararon documentos sobre política monetaria, a fin de informar a la sociedad sobre la forma en que el BCCh operaba para cumplir con su objetivo de estabilidad de precios (Massad 2014; BCCh 2007), para facilitar así la comprensión de sus decisiones.

En los últimos años, el Banco Central, al igual que otros bancos centrales del mundo, ha hecho avances importantes en seguridad informática, la gestión de riesgos, el control de gestión y la transparencia. Para este propósito, se estudiaron experiencias internacionales de organización de otros bancos centrales e instituciones similares, estudios tendientes a desarrollar un proyecto destinado a lograr un cumplimiento más eficiente de los objetivos establecidos en la Ley Orgánica Constitucional y a fortalecer las labores de control interno (Corbo 2014).

Además, para fortalecer la autonomía, el banco ha hecho en los últimos diez años avances importantes en el acceso a la información, en materia de transparencia y en las comunicaciones, tanto internas como externas.

En paralelo, el Banco ha hecho notables esfuerzos en robustecer el trabajo técnico en el área macrofinanciera, con el fin de cumplir en mejor forma los objetivos fundamentales de estabilidad de precios y el normal funcionamiento de los pagos internos y externos. De esta forma, se actualizó el marco de política monetaria, explicitando una meta de inflación de tres por ciento con un rango de tolerancia de uno por ciento y perfeccionando la operatoria de un esquema de metas de inflación flexible con flotación cambiaria, donde las intervenciones se hacen sólo en casos excepcionales, los que se encuentran explicitados en su marco de política.

El trabajo técnico del banco, fruto del esfuerzo de un equipo profesional de excelencia, le ha permitido alcanzar un alto grado de reconocimiento, tanto a nivel nacional como internacional, el que se ve reflejado en el volumen, la calidad y el uso que se hace de sus publicaciones, $\mathrm{y}$ en el prestigio que ha alcanzado su conferencia anual. 
El éxito del Banco Central para llevar la inflación de tendencia a la meta junto con el fortalecimiento de la autonomía le han permitido en años recientes implementar políticas para llevar a cabo cambios importantes en precios relativos, sin alejar la inflación de tendencia de su meta (véanse Gráfico 1 y Gráfico 2).

En años recientes, el Banco Central ha continuado fortaleciendo su marco de políticas, dándole más peso a su capacidad de respuesta frente a crisis financieras globales (De Gregorio 2014). Gracias a su marco institucional y a la contribución de muchos, el BCCh es hoy una historia de éxito que le ha permitido al país alcanzar y mantener la estabilidad de precios, condición necesaria para un crecimiento alto y sostenido. Ello ha posibilitado un gran avance en materia de estabilidad macrofinanciera y, a la vez, contar con una poderosa herramienta anticíclica para intentar mantener el producto en las cercanías del producto potencial, lo que ha permitido al país superar la gran crisis financiera sin consecuencias adversas para su sistema financiero y la población en general. Sin duda, estos logros le han merecido al Banco Central el respeto de la sociedad, lo que a su vez ha servido para fortalecer su autonomía.

A estos logros han contribuido el marco de políticas utilizado por el Banco (BCCh 2007), la fortaleza de las finanzas públicas amparada

\section{Gráfico 1. INFLACIÓN EN CHILE 1950-2014*}

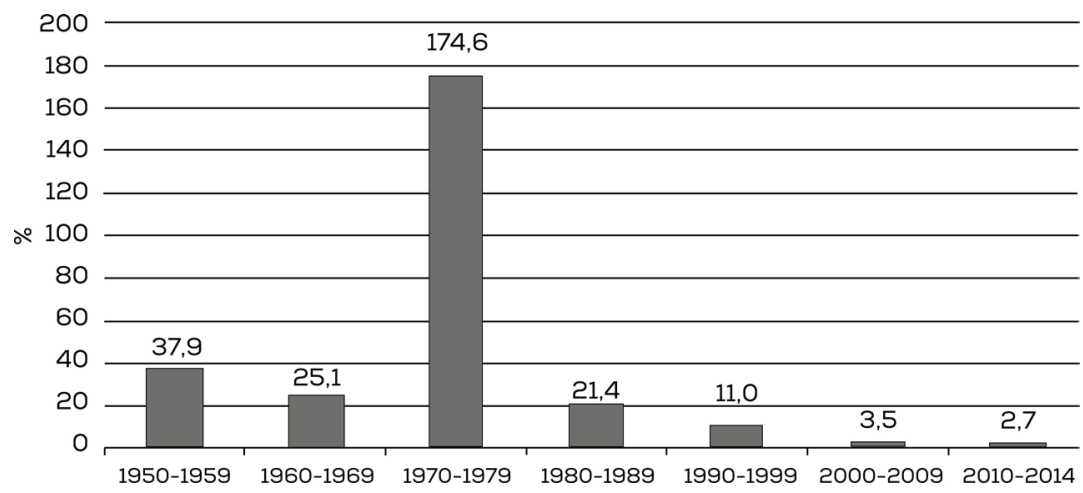

* Promedio simple de periodos indicados.

Fuente: Elaboración propia en base a World Development Indicators, Banco Mundial. 
Gráfico 2. INFLACIÓN Y META EN CHILE 1990-2014

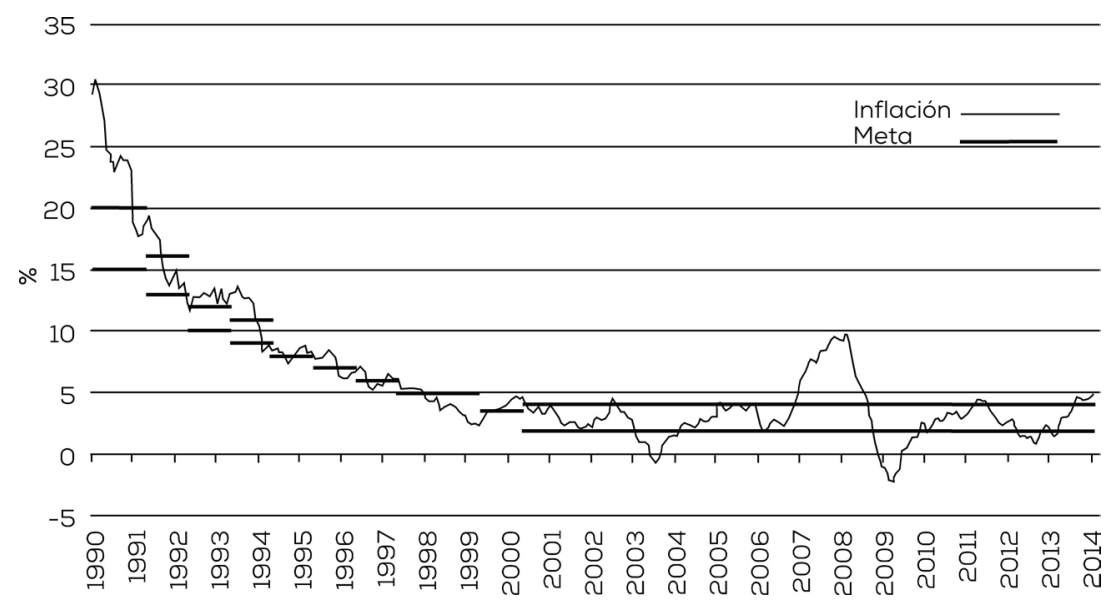

Fuente: Elaboración propia en base a Céspedes y Soto (2006), y Banco Central de Chile.

en una regla fiscal acíclica (CADPF 2011) y la estabilidad financiera, esta última apoyada por una apropiada regulación y supervisión financiera. Como lo ilustra esta narrativa, el Banco Central que tenemos ahora es el resultado de 25 años de aprendizaje, tanto de la experiencia interna como externa. En esto ha jugado un rol central el consejo del Banco y el profesionalismo de su personal.

\section{OPORTUNIDADES A LUZ DE LA AUTONOMÍA DEL BANCO CENTRAL DE CHILE}

Las instituciones autónomas son un elemento clave para una democracia. Junto con el poder ejecutivo, legislativo y judicial, las instituciones autónomas proveen un adecuado sistema de checks and balances y mejoran la rendición de cuentas. El término autonomía hace alusión a la necesidad de que algunas instituciones cuenten con las herramientas para salvaguardar sus decisiones de influencias políticas indebidas, dotándolas de independencia política y operativa.

Las condiciones básicas para que las instituciones autónomas operen libre y eficazmente son: i) independencia financiera, que asegure que puedan disponer de los recursos humanos y materiales para cumplir 
su mandato; ii) independencia de nombramiento y permanencia, que asegure un staff de probada y reputada calidad profesional, y un consejo con permanencia fija, que no esté sujeto a remociones arbitrarias por parte del poder ejecutivo o legislativo; iii) independencia en la toma de decisiones, que asegure que el ejecutivo no tenga influencia en las recomendaciones hechas por estas instituciones; y iv) adecuada y frecuente rendición de cuentas, para responder así a la responsabilidad encomendada por la sociedad.

En Chile, a pesar de los avances en la construcción de instituciones, hay otras áreas en donde el país también se puede beneficiar de mejoras institucionales orientadas a aumentar la autonomía de órganos del Estado. Un área de particular relevancia para el país, y que puede beneficiarse de la experiencia de autonomía del Banco Central de Chile, es la producción y análisis de estadísticas oficiales. Otra área de mejora institucional clave para el país es la creación de una Comisión de Productividad, cuyo foco debería estar en reformas que estimulen el crecimiento de la productividad y el bienestar de los ciudadanos.

Un Instituto Nacional de Estadísticas y una Comisión de Productividad, que cuenten con un nivel aceptable de autonomía y gobiernos corporativos colegiados de alto nivel técnico, pueden contribuir a mejorar la calidad y confiabilidad de las estadísticas y a generar propuestas de políticas con un alto estándar técnico, $y$, tomando en consideración los problemas de economía política asociados a ellas, puede también contribuir a diseñar y sostener el proceso de reformas, de tal manera que la economía chilena avance en la implementación de reformas bien diseñadas y discutidas por la sociedad con miras a lograr el desarrollo.

Ahora analizaremos estas dos áreas de reformas en más detalle.

\subsection{Instituto Nacional de Estadísticas}

De acuerdo a la Comisión de Estadísticas de Naciones Unidas, las estadísticas oficiales constituyen un bien público y son un elemento indispensable para la rendición de cuentas en una sociedad democrática. Ellas deben procurar proveer al gobierno, a la economía y a los ciudadanos información confiable sobre la situación económica, social, demográfica y ambiental del país. Para cumplir estos fines, los organismos oficiales de estadísticas han de recolectar y producir, en 
forma imparcial, cifras de comprobada calidad para ser utilizadas en el diseño y evaluación de políticas públicas y, en general, para la toma de decisiones de los distintos miembros de la sociedad (UN 2013). Para cumplir con estos objetivos, es fundamental fortalecer el actual Instituto Nacional de Estadísticas (INE), dándole autonomía y robusteciendo su carácter técnico. La autonomía de esta institución debería, por supuesto, incluir la designación de sus autoridades, su administración y financiamiento. Un INE autónomo, y con los recursos necesarios para atraer profesionales de alto nivel técnico, permitiría asegurar que las estadísticas generadas estén libres de interferencia política y su calidad sea reconocida por toda la sociedad. Esta independencia del gobierno sería especialmente importante en la elaboración de las cifras utilizadas en la evaluación de programas públicos.

Toda propuesta para fortalecer la institucionalidad del INE debiera incluir: i) aumentar su capacidad técnica; ii) la creación de un gobierno corporativo colegiado, compuesto por profesionales de reconocida capacidad técnica, con distintas visiones sobre la sociedad y con objetivos claros de las principales estadísticas que se debieran recopilar y producir; y iii) un adecuado presupuesto, que le permita contratar al personal y los servicios necesarios para cumplir con sus objetivos. Un INE fortalecido debiera, también, apoyar técnicamente a otras reparticiones públicas encargadas de recolectar y generar estadísticas sectoriales. Muy buenas experiencia internacionales en esta área son las oficinas de estadísticas de Canadá, Australia, Dinamarca y Noruega.

\subsection{Comisión de Productividad}

La creación de una Comisión de Productividad (en adelante CP) es otra área de mejora institucional clave para el país, ahora que los beneficios de las reformas de primera generación ya están maduros. Su objetivo debiera ser el diseño y evaluación de reformas que apunten a estimular el crecimiento de la productividad y el bienestar de los ciudadanos. Como el crecimiento económico no es la única dimensión relevante del bienestar, los impactos sociales, regionales y medioambientales también deberían ser parte integral del diseño y de la evaluación de políticas llevadas a cabo por la CP. La reciente agenda de productividad anunciada por el Ministerio de Economía, que incluye la creación de una $\mathrm{CP}$, puede ser una instancia propicia para avanzar en esta dirección. 
Existen experiencias exitosas en cuanto al desempeño de una CP. De hecho, Australia y Nueva Zelandia, dos países ricos en recursos naturales, integrados al mundo y con economías de mercado que han podido alcanzar el desarrollo, crearon CPs para perseguir los objetivos señalados en el párrafo anterior. En estos países, las CPs son organismos autónomos del Estado, cuya independencia y mandatos están establecidos en la ley (similar a la operación del BCCh). En dichos países, las CPs analizan y evalúan las opciones de políticas públicas en las áreas solicitadas por el poder ejecutivo, y también en las que surgen de su propia iniciativa. Por esta razón, la autonomía de esta institución es fundamental para poder cumplir con su mandato.

Las CPs de Australia y Nueva Zelandia cuentan con dos elementos claves para el buen diseño de reformas: un equipo técnico permanente y altamente calificado (que no varía en función de los cambios de administración), y un proceso de consulta ciudadana. Este mecanismo incluye un exhaustivo debate público a través de audiencias, talleres y foros, donde se publican y actualizan los informes y resultados preliminares de las opciones de políticas públicas en áreas específicas en las que existen oportunidades para mejorar la productividad y el bienestar. Estas instancias entregan la oportunidad para que los diferentes puntos de vista sean escuchados y para que todas las partes interesadas y potencialmente afectadas puedan participar en el diseño de una reforma.

El trabajo de la CP concluye con la publicación de su recomendación final, y aunque el gobierno no está obligado a hacerla suya, en la práctica, la mayoría de las recomendaciones son eventualmente aceptadas. Además, la alta calidad de los estudios, que reúnen el contenido técnico de las reformas con las visiones de los actores interesados, fortalece el proceso deliberativo de éstas en el Congreso, a pesar de que la $\mathrm{CP}$ no participa en el debate de los proyectos presentados por el ejecutivo.

En Australia, la CP también evalúa las regulaciones y reformas recomendadas por ella, después de haber sido implementadas, lo que ayuda a sostener el complejo proceso político de elaboración de reformas y refuerza la credibilidad de la comunidad en la CP. De hecho, según la OCDE, la CP de Australia es la institución más efectiva en el diseño y sostenibilidad de las reformas para promover el desarrollo económico y social de su país (OECD 2010). Este éxito se debe a que, en parte, las reformas llegan mucho mejor preparadas al proceso legislativo. 
Con todo, parte importante de la exitosa labor del BCCh, y que se puede extender a otras instituciones, radica en su autonomía y su gobierno corporativo de alto nivel técnico. De esta forma, un INE y una $\mathrm{CP}$ que cuenten con ambos aspectos, además de los recursos necesarios para atraer profesionales de altos estándares profesionales, contribuirán a generar mejor información, el primero, y a diseñar y sostener el proceso de reformas, la segunda, de tal forma que permitan a Chile avanzar en la implementación de reformas bien diseñadas y discutidas por la sociedad con miras a lograr el desarrollo.

\subsection{Otras áreas para mejorar}

Pero también hay otras áreas donde nos podemos beneficiar con fortalecimiento institucional. En particular, me refiero a la creación de un Consejo Fiscal independiente del ejecutivo, que analice en forma autónoma las cuentas fiscales y la solvencia de proyectos de ley como de desarrollos demográficos y tendencias globales y nacionales. Una propuesta en esta dirección ya la hizo el comité asesor para el diseño de una política fiscal de balance estructural de segunda generación para Chile. La propuesta de ese comité fue la creación de un Consejo Fiscal, con un gobierno corporativo de tres consejeros elegidos entre especialistas académicos o de alta experiencia en materias fiscales, propuestos por el ejecutivo y ratificados por el Congreso, con períodos de permanencia traslapados, independientes del ciclo presidencial. ${ }^{1} \mathrm{Se}$ recomendó, también, que el ejercicio de la presidencia implique un cargo de tiempo completo mientras que los restantes miembros trabajarían a tiempo parcial (verificando que el cargo no entre en conflicto con sus restantes actividades). A su vez, el presidente sería seleccionado entre los consejeros por el Presidente de la República.

\section{CONCLUSIONES}

En el último cuarto de siglo, Chile ha implementado importantes reformas institucionales que han permitido fortalecer su estabilidad macroeconómica, construir un sistema financiero bien regulado y super-

${ }^{1}$ A modo de ejemplo, la primera vez podrían ser elegidos por dos, cuatro y seis años respectivamente, para que luego, en régimen, cada miembro sea elegido por seis años. Para detalles sobre esta propuesta ver CADPF (2011). 
visado y crear una economía más competitiva y abierta al mundo. Estas reformas le permitieron a Chile dar un salto en su desarrollo, con un aumento notable en su nivel de ingreso per cápita y con grandes mejoras en la reducción de la pobreza y en una serie de indicadores sociales, lo que ha hecho del país un ejemplo para la región y el mundo.

En este artículo he expuesto una reforma institucional que, a mi juicio, ha sido muy importante para la calidad de la política económica de Chile: la autonomía del Banco Central.

En cuanto a oportunidades para robustecer instituciones en otras áreas, tanto el fortalecimiento del Instituto Nacional de Estadísticas como la creación de una Comisión de Productividad autónoma requieren que se cumplan tres tipos de independencia: en lo financiero; en el nombramiento y permanencia de sus consejeros; y en la toma de decisiones técnicas. En el caso particular del INE, se necesita aumentar su capacidad técnica, mejorar su gobierno corporativo y su presupuesto. Por su parte, la creación de una CP debe enfocarse en diseñar, analizar y legitimar reformas que estimulen el crecimiento de la productividad y, por lo tanto, del bienestar de la población.

\section{REFERENCIAS BIBLIOGRÁFICAS}

Acemoglu, Daron \& James Robinson. 2012. Why Nations Fail. New York: Crown Publishing Group.

Banco Central de Chile (BCCh). 2007. La política monetaria del Banco Central de Chile en el marco de metas de inflación. http://www.bcentral.cl/publicaciones/ politicas/pdf/politica_monetaria_metas.pdf/

Bianchi, Andrés. 2014. "La independencia del Banco Central: los años iniciales." Conferencia en seminario "The Role of Central Banks in Modern Times: Twenty-Five Years into the Central Bank of Chile's Independence." 24 de octubre. http:/www.bcentral.cl/conferencias-seminarios/otras-conferencias/ pdf/Conference24102014/1-\%20Andres\%20Bianchi.pdf/

Comité Asesor para el Diseño de una Política Fiscal de Balance Estructural de Segunda Generación para Chile (CADPF). 2011. "Propuestas para el perfeccionamiento de la regla fiscal. Informe final." http://www.dipres.gob. cl/572/articles-76544_doc_pdf.pdf/

Corbo, Vittorio. 2014. "Algunas reflexiones sobre mi período en la presidencia del Banco Central de Chile." Conferencia en seminario "The Role of Central Banks in Modern Times: Twenty-Five Years into the Central Bank of Chile's Independence." 24 de octubre. http://www.bcentral.cl/ 
conferencias-seminarios/otras-conferencias/pdf/Conference $24102014 / 4 \% 20$ -\%20Vittorio\%20Corbo.pdf/

De Gregorio, José. 2014. “A 25 años de la autonomía del Banco Central: algunas lecciones de política". Conferencia en seminario "The Role of Central Banks in Modern Times: Twenty-Five Years into the Central Bank of Chile's Independence." 24 de octubre. http://www.bcentral.cl/conferenciasseminarios/otras-conferencias/pdf/Conference24102014/5\%20-\%20Jose \%20 De\%20Gregorio.pdf/

Gredig, Fabián, Klaus Schmidt-Hebbel \& Rodrigo O. Valdés. 2008. "El horizonte de la política monetaria en Chile y otros países con metas de inflación." Economía Chilena 11(1): 5-27. http://www.bcentral.cl/eng/studies/economiachilena/2008/apr/v11n1abr2008pp5-27.pdf/

Massad, Carlos. 2014. "25 Years into the Central Bank of Chile's Independence." Conferencia en seminario "The Role of Central Banks in Modern Times: Twenty-Five Years into the Central Bank of Chile's Independence." 24 de octubre. http://www.bcentral.cl/conferencias-seminarios/otras-conferencias/ pdf/Conference24102014/3\%20-\%20Carlos\%20Massad.pdf/

Mishkin, Frederic \& Klaus Schmidt-Hebbel. 2007. "Monetary Policy under Inflation Targeting: An Introduction." En Central Banking, Analysis, and Economic Policies Book Series. Volume 11: Monetary Policy Under Inflation Targeting, editado por Frederic S. Mishkin \& Klaus Schmidt-Hebbel. Santiago de Chile: Central Bank of Chile. http://www.bcentral.cl/eng/studies/centralbanking/v11.htm/

Olson, Mancur. 1984. The Rise and Decline of Nations: Economic Growth, Stagflation, and Social Rigidities. New Have: Yale University Press.

Organization for Economic Cooperation and Development (OECD). 2010. "Opening Markets to Competition." En Making Reform Happen: Lesson from OECD Countries. OECD Publishing. http://www.oecd.org/site/ sgemrh/46159078.pdf/

Soto, Claudio \& Luis F. Céspedes. 2006. "Credibility and Inflation Targeting in Chile." Working Papers Central Bank of Chile 408. Central Bank of Chile.

United Nations (UN). 2013. "Fundamental Principles of Official Statistics. Good Practices on National Official Statistics. United Nations Statistics Division." http://unstats.un.org/unsd/dnss/gp/FP-New-E.pdf/

Zahler, Roberto. 2014. "25 años de independencia del Banco Central.” Conferencia en seminario "The Role of Central Banks in Modern Times: Twenty-Five Years into the Central Bank of Chile's Independence." 24 de octubre. http://www.bcentral.cl/conferencias-seminarios/otras-conferencias/pdf/ Conference24102014/2\%20-\%20Roberto\%20Zahler.pdf/. EP 